\title{
COMPARATIVE STUDY OF MEASURING PHYSICAL ACTIVITY AMONG SPORT STUDENTS
}

\section{Jordan Donev, Stoyan Andonov, Stefka Djobova, Jordan Donev, Oleg Hristov, Ivelina Kirilova, Stoyan Bahchevanski, Rumen Iosifov, Velichka Aleksandrova, Bogomil Angelov \& Natalia Stoyanova}

\author{
National Sports Academy "Vassil Levski”, Sofia, Bulgaria
}

\begin{abstract}
The aim of this pilot study is to research the relation between subjective and objective methods of measuring physical activity. Methods: Data have been collected by self-administrated International Physical Activity Questionnaire Short Form and measured through accelerometer Axivity AX3. Initially 124 students randomly selected participated - 32 females and 92 males, aged 18 - 40 years. Accelerometers Axivity AX3 were worn during seven consecutive days and at the end of the period International Physical Activity Questionnaire Short Form was completed. In order to compare the data collected from the IPAQ-SF and AX3, we used the following statistical methods: Quantitative Exploratory data analysis, Spearman Correlation Coefficient, Wilcoxon signed rank tests. Results: There is no correlation between data collected from IPAQ and AX3. A value given by a person in the IPAQ is not related to the actual AX3 value. There is weak correlation between intensities regarding differences - IPAQ - AX3. Conclusion: People are a bit consistent in their errors between intensities. As their error for one intensity increases, so does their error of the other intensities. However, the data that IPAQ-SF yields, when compared to objectively collected one is very inaccurate. That being said, we would not recommend using IPAQ-SF as an independent method of measuring physical activity.
\end{abstract}

Key words: accelerometry, questionnaire, subjective, objective

\section{INTRODUCTION}

Benefits of physical activity (PA) are well known and scientifically evidence based. No matter of that, for the $6 \%$ of the world population physical inactivity is the leading factor for mortality (WHO, 2009). During the last decades sedentary lifestyle turns into an epidemic.

Country's health systems, policymakers, stakeholders and society become aware that it is necessary to be taken actions immediately. One important step was undertaken in May 2005 when The European network for the promotion of health-enhancing physical activity (HEPA Europe) was launched as a new international, collaborative initiative assisted by WHO/Europe: „Health-enhancing physical activity". HEPA is "any form of physical activity that benefits health and functional capacity without undue harm or risk". The overall vision of this initiative is to achieve better health through PA among all people in Europe (WHO, 2005). According to WHO for "adults aged 18-64 PA should be at least 150 minutes of moderate-intensity aerobic PA throughout the week or at least 75 minutes of vigorous-intensity aerobic PA throughout the week or an equivalent combination of moderate - and vigorous-intensity activity..." (WHO, 2010) But following these or any recommendations and measuring the actual levels of PA of the population turns into a challenge for many country's health systems. Through the years many efforts were made for development of reliable and valid instruments for estimation of PA levels. As a leading organization in the field of human health, WHO developed in the distant 1998-year International Physical Activity Questionnaire (IPAQ) in two form - long and short. It has been translated in more than 21 languages and become one of the wide spreaded questionnaire for surveillance of PA and to facilitate global comparison (Craig et ai, 2003). Nowadays there is a wide range of available common instruments to measure PA include self-reported questionnaires, observation heart rate monitoring, calorimetry, movement/activity sensors and ect. Still common applied by national health systems subjective method is self-reported questionnaires as a means of data collection. They are preferred instrument because of their rapidness and cost efficiency, but many researchers have doubts about their reliability. Following this trends Bulgarian health system also collect data with the similar instruments. 
As an alternative method for more precise measurement of PA and achieving high degree of standardization in PA health related levels definition and assessment the popularity of accelerometers has grown recently. They are described as not usually practical in large-scale cohort studies due to their high costs and instead questionnaires are frequently used to obtain physical activity data, they have obvious advantages: objective measurement, small and unobtrusive size. The accelerometer is often used as the gold standard against which self-report questionnaires are compared (Hagströmer et al, 2010). Both methods have their strengths and weaknesses, but more evidence base researches are needed in the field.

\section{METHODS}

Participants in our pilot study were recruited from the first- and second-year students in sport and physical education studying at the National sports academy in Sofia, Bulgaria. Initially 124 students randomly selected participated - 32 females and 92 males. Students were informed of the study and asked for their willingness to participate. Participants were instructed to wear continuously the accelerometers AX3 during 7 consecutive days. The AX3 were worn attached to the wrist. At the end of the period when they give back the AX3, participants completed the IPAQ-SF.

For the experiment, a group of 98 students ( $28 \mathrm{fe}-$ males and 70 males) was formed which completed both accelerometry and questionnaire.

After the preliminary data analysis, we applied exclusion criteria encompassing all participants who were worn the AX3 devices less than 24 hours. Seven persons did not meet these criteria. After the initial cleaning, our data consists of 85 volunteers who have completed 2 tests, measuring time spent in 3 intensities of PA for a period of 6 days. However, after dropping NAN - values, separately for each level of PA intensity, we are left with 77 values for light 70 for moderate and 81 for vigorous PA. NAN values represent 'no information given' in the IPAQ. We drop these values separately for each intensity, since dropping all participants with a NAN value, will result in losing nearly $30 \%$ of our data. Throughout our analysis, we will refer to IPAQ and AX3 as tests, Physical activity as PA and light, moderate and vigorous as intensities. Where relevant, when referring to IPAQ and AX3 we mean the data provided by the corresponding test. All our data is measured in minutes/24h, except if specified otherwise.

All statistical results were acquired using Python 3.7. for Windows 10. In order to compare the data collected from the IPAQ and AX3, we used the following statistical methods: Quantitative EDA, ECDF and normal CDF, Spearman Correlation Coefficient, Wilcoxon signed rank tests.

\section{Data Analysis}

In the following sections, we will first compare the sets of data provided by the two tests. Next, we will inspect the differences between values from the IPAQ and the AX3 tests for each volunteer. Then, having completed our exploratory analysis, we are going to test if data from the two tests comes from similar distributions and weather differences (IPAQ - AX3) have a positive or negative median. In the last section, we will focus on correlation between the two tests and between differences (IPAQ - AX3) for each intensity.

Exploratory data analysis (EDA). Figure 1 and Table 1 represents data grouped by intensity and test.



Figure 1. Boxplot grouped by intensity and test 
Table 1. Data grouped by intensity and test

\begin{tabular}{|l|c|c|c|c|c|c|}
\hline & Light AX3 & $\begin{array}{c}\text { Light } \\
\text { IPAQ }\end{array}$ & Moderate AX3 & Moderate IPAQ & $\begin{array}{c}\text { Vigorous } \\
\text { AX3 }\end{array}$ & $\begin{array}{c}\text { Vigorous } \\
\text { IPAQ }\end{array}$ \\
\hline Count & 77 & 77 & 70 & 70 & 81 & 81 \\
\hline Mean & 69.6 & 112.1 & 203.8 & 95.5 & 10.6 & 175.2 \\
\hline Std & 32.5 & 92.2 & 101.8 & 91.4 & 10.9 & 145.3 \\
\hline Min & 1 & 3 & 2 & 6 & 0 & 9 \\
\hline $\mathbf{2 5 \%}$ & 52 & 43 & 147 & 34 & 2 & 77 \\
\hline $\mathbf{5 0 \%}$ & 73 & 64 & 219.5 & 51 & 8 & 129 \\
\hline $\mathbf{7 5 \%}$ & 89 & 180 & 277.8 & 132.8 & 14 & 270 \\
\hline Max & 138 & 480 & 370 & 360 & 65 & 630 \\
\hline
\end{tabular}

From Figure 1 and Table1, we see that although PA and (4.5hrs, 10.5hrs) for Vigorous PA from the IPAQ and AX3 have similar medians for Light PA, IPAQ look extreme, when considered in reality. the standard deviation and mean of IPAQ are much We notice that IPAQ yields higher values for Vigorhigher. Seeing even more notable differences in ous PA and AX3 - for Moderate PA. Thus, we would the other two intensities, we can suggest that the like to compare the sum of time spent in these intwo tests provide data with different distributions. tensities for the two tests, seeing it as Higher (intenAlso, upper quarter ranges of (3hrs, 8hrs) for Light sity) PA - Figure 2.

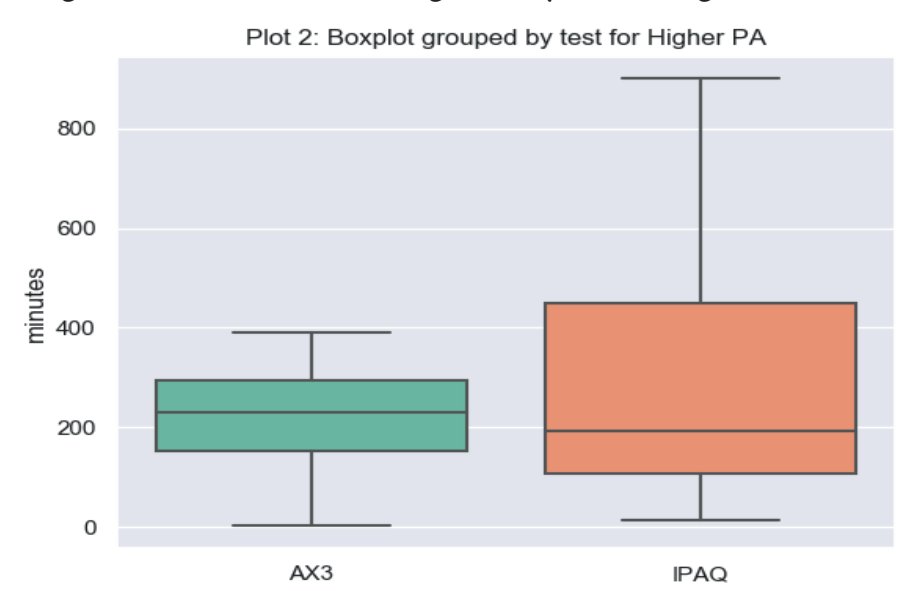

Figure 2. Boxplot grouped by test - IPAQ and AX3 for Higher PA

Our second boxplot show us differences between the two tests (for Higher PA) similar to the ones we observed for Light PA.

In the last part of our EDA, we will inspect the sets of differences, between values from IPAQ and val- ues from $\mathrm{AX} 3$, for each volunteer, grouped by intensity. That is, assuming that $\mathrm{AX} 3$ gives us the true values, we want to investigate the errors (IPAQ AX3) that people have made. These differences are represented on Figure 3 by boxplot.

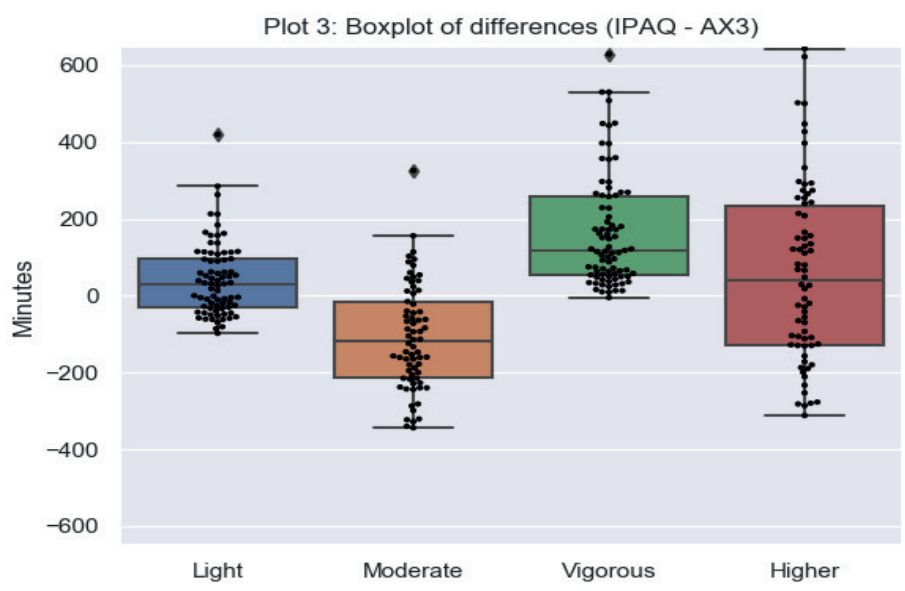

Figure 3. Boxplot of Differences between values from IPAQ and values from AX3 
From our third boxplot, we observe that more than $75 \%$ of our data for Moderate PA is negative and nearly all our data for Vigorous PA is positive. However, the medians for Light and Higher PA are slightly greater than 0 , with data being distributed on both sides of the horizontal axis, thus leaving us uncertain of the sign of the difference between IPAQ and AX3 for these intensities.

After our EDA, following questions were shifted - first, do the two tests provide data with similar distributions, for each PA intensity and second, are the differences (IPAQ - AX3) mostly positive or negative (for each intensity)? We can answer both of these questions using the Wilcoxon Signed-Rank Test (WSRT).

Here, we test the null hypotheses that AX3 and IPAQ come from the same distribution, for each intensity. We also obtain a $95 \%$ confidence interval for and an estimate of the median of the differences. Results are shown on Table 2.

Table 2. Wilcoxon Signed Rank Test

\begin{tabular}{|l|l|l|l|l|}
\hline & \multicolumn{1}{|c|}{ Light } & \multicolumn{1}{c|}{ Moderate } & \multicolumn{1}{c|}{ Vigorous } & \multicolumn{1}{c|}{ Higher } \\
\hline Statistic & $\mathrm{V}=2088.5$ & $\mathrm{~V}=326$ & $\mathrm{~V}=3320$ & $\mathrm{~V}=1525.5$ \\
\hline P-value & 0.001212 & $8.29 \mathrm{e}-08$ & $5.67 \mathrm{e}-15$ & 0.09828 \\
\hline 95\% conf. int. & {$[14,55.5]$} & {$[-146.5,-77.5]$} & {$[111.0,180.5]$} & {$[-8,107]$} \\
\hline Median estimate & 35 & -113 & 145 & 48 \\
\hline
\end{tabular}

P-values for the first three intensities in the table level 0.1 means that there is little or no evidence are extremely low, thus firmly rejecting the null hy- against the null hypothesis. Our $95 \%$ confidence inpothesis that IPAQ and AX3 come from the same distribution. We can see that the confidence interval for the median of the differences is positive for Light and Vigorous PA and negative for Moderate PA.

For the Higher intensity, a p-value significant at terval is $(-8,107)$ here. However, a $90 \%$ confidence interval will be strictly positive.

Before our final discussion, we will focus on correlation, first looking at correlation between IPAQ and AX3. Below, on Figure 4 we have four scatter plots for each intensity.
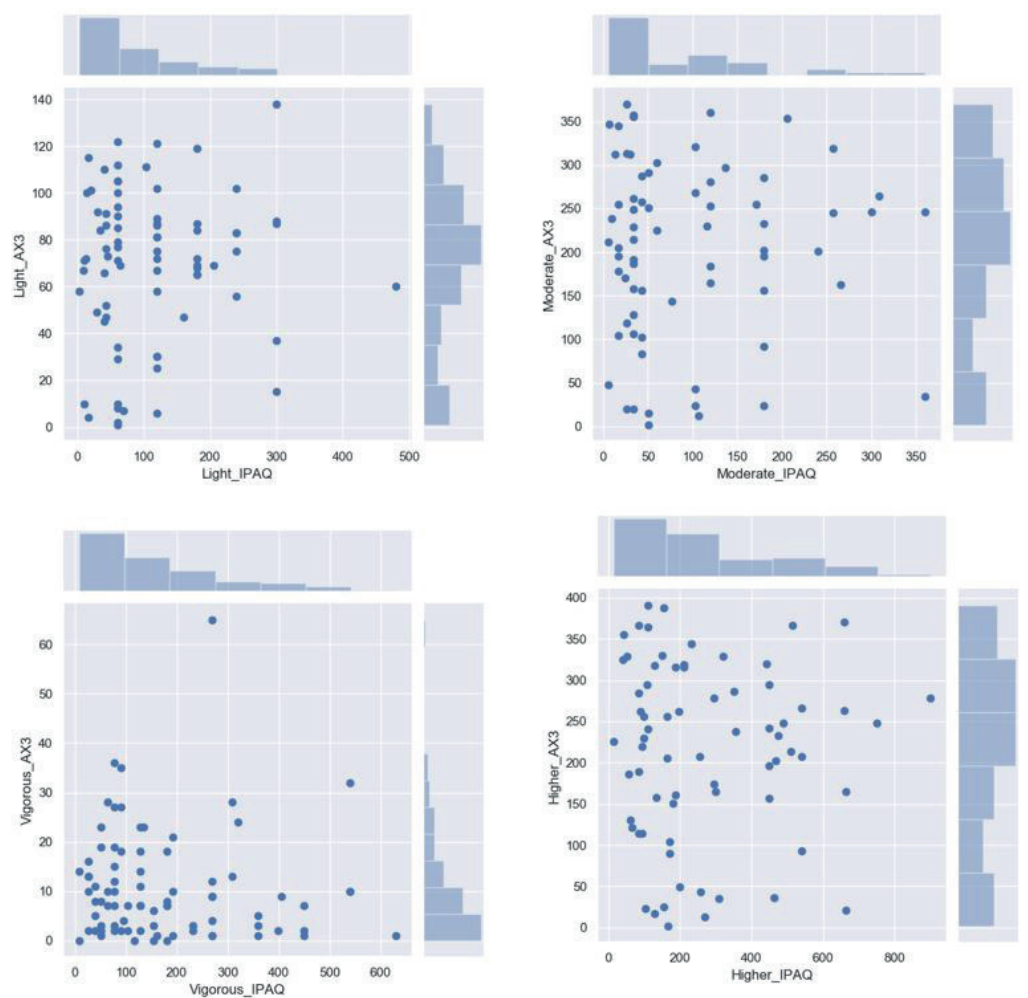

Figure 4. Scatter plots of correlation between each intensity - AX3 and IPAQ

Table 3 contains Spearman coefficients for each in- there is no correlation between the two tests. tensity and p-values for the null hypotheses that 
Table 3. Spearman coefficients for each intensity and p-values - null hypotheses

\begin{tabular}{|l|l|l|l|l|}
\hline & \multicolumn{1}{|c|}{ Light } & \multicolumn{1}{c|}{ Moderate } & \multicolumn{1}{c|}{ Vigorous } & \multicolumn{1}{c|}{ Higher } \\
\hline Spearman's $\rho$ & 0.027 & -0.012 & -0.128 & -0.047 \\
\hline P-value & 0.814 & 0.92 & 0.255 & 0.702 \\
\hline
\end{tabular}

Obtaining Spearman coefficients close to zero and ferences (IPAQ - AX3) in each intensity. In other p-values much greater than 0.1 , we can conclude that there is no monotonic relationship between the two tests (i.e. a value provided by a volunteer in the IPAQ is not related to the actual AX3 value). Finally, we test for the correlation between the dif- each pair of intensities).
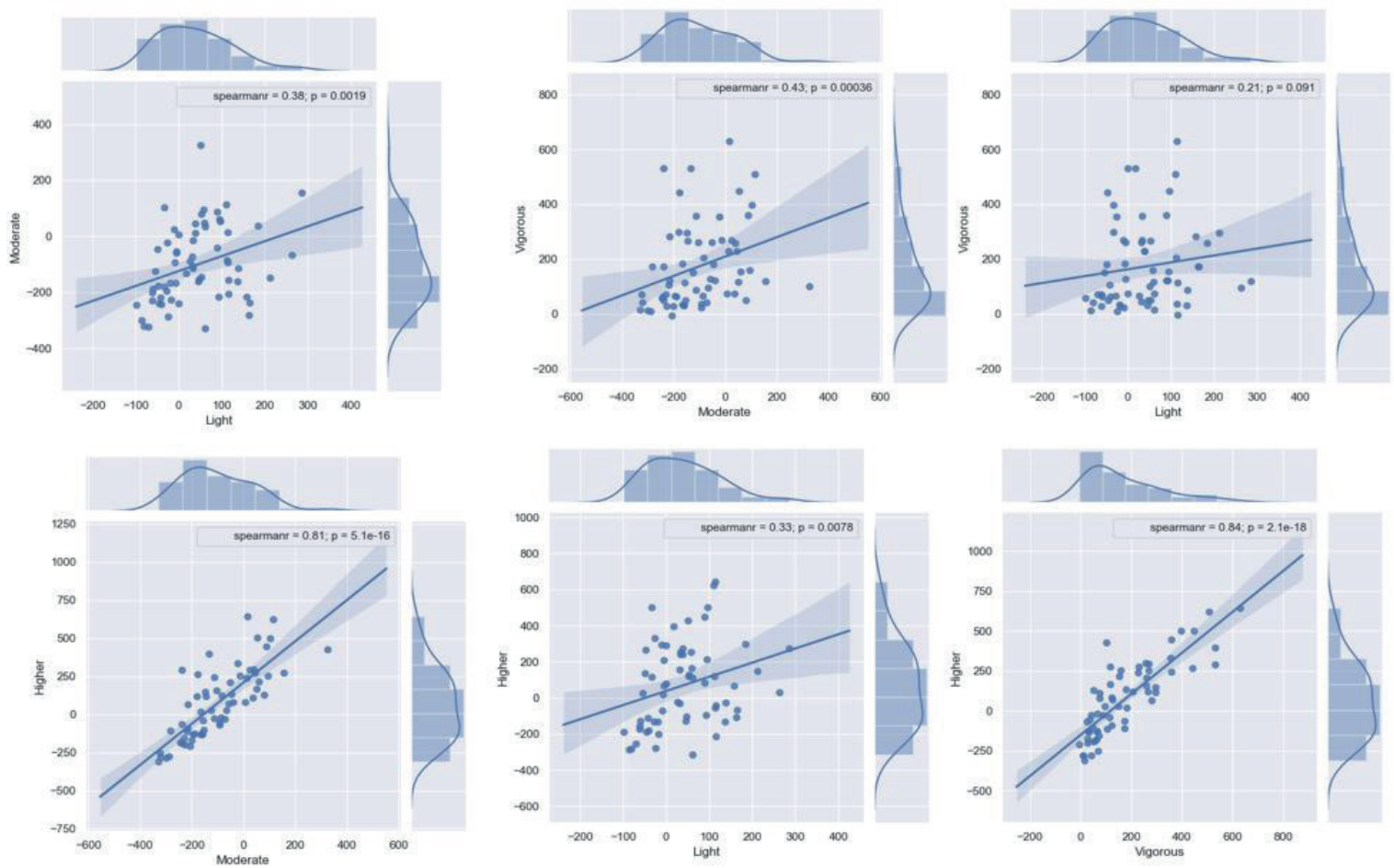

Figure 5. Scatter plots of correlation between the differences (IPAQ - AX3) in each intensity

Table 4 contains information similar to that of Ta- Moderate, Vigorous or Higher in this section, we ble 3. For our convenience, when referring to Light, mean the data for the corresponding intensity.

Table 4. Correlation between the differences (IPAQ - AX3) in each intensity

\begin{tabular}{|c|c|c|c|c|c|}
\hline & & Light & Moderate & Vigorous & Higher \\
\hline \multirow{2}{*}{ Light } & Spearman's $\rho$ & 1 & & & \\
\hline & P-value & \begin{tabular}{|l|}
0.0 \\
\end{tabular} & & & \\
\hline \multirow{2}{*}{ Moderate } & Spearman's $\rho$ & 0.382 & 1 & & \\
\hline & P-value & 0.00187 & 0.0 & & \\
\hline \multirow{2}{*}{ Vigorous } & Spearman's $\rho$ & 0.213 & 0.433 & 1 & \\
\hline & P-value & 0.09091 & 0.00036 & 0.0 & \\
\hline \multirow[b]{2}{*}{ Higher } & Spearman's $\rho$ & 0.330 & 0.810 & 0.844 & 1 \\
\hline & P-value & \begin{tabular}{|l|}
0.00778 \\
\end{tabular} & $5.104 \mathrm{e}-16$ & $2.11 \mathrm{e}-18$ & 0.0 \\
\hline
\end{tabular}

The greatest p-value from Table 4 (0.091) is the one for the test of no correlation between Light and Vigorous. The corresponding Spearman $\mathrm{r}$ is 0.213 . This tells us that there is weak and not that significant monotonic relation between the two variables in question. The pairs Light-Moderate, Light-Higher and Moderate-Vigorous all have statistically significant correlations (low p-values), although not that strong, since their Spearman coefficients are all less than 0.5. 
Greatest correlation can be seen between Higher and Moderate/Vigorous intensities, observing extremly low p-values and coefficients greater than 0.8 . However, this is something that we would expect since Higher is the sum of Moderate and Vigorous.

\section{DISCUSSION}

Having worked with both the International Physical Activity Questionnaire Short Form (IPAQ-SF) and the AX3 accelerometer, we would like to make a few notes regarding the two tests.

IPAQ-SF - First, it is hard for people to remember what physical activities they have performed during the past week as well as the intensities of their activities. We get unrealistic values that makes us think that questions are not interpreted properly by some people. A lot of people decide not to answer certain questions, which results in NAN-values, which are useless for our analysis.

Axivity AX3 - Many people didn't wear their accelerometer all the time, resulting in data loss.

Considering accelerometers and the way they collect data, we are left uncertain of their accuracy regarding types of intensities, for specific exercises (e.g. pullups). However, this is something beyond the aim of this research, which has to be studied further. From our point of view, accelerometers provide us with objectively collected data which is accurate enough for the purpose of this study.

From our Exploratory Data Analysis, we observed that IPAQ has a much higher standard deviation than AX3 for most intensities. It provides us with values from a bigger range.

Performing statistical tests and assuming AX3 gives us the true values, we found out that IPAQ gives values from a different distribution. Time suggested by people in the IPAQ is on a different scale compared to the time collected by the accelerometers. People are extremely inaccurate in recognizing types of intensities. They tend to overestimate themselves for Light Vigorous and Higher PA and underestimate themselves regarding Moderate PA. There is no correlation between IPAQ and AX3. A value given by a person in the IAPQ is not related to the actual AX3 value. There is weak correlation between intensities regarding differences (IPAQ - AX3). People are a bit consistent in their errors between intensities. As their error for one intensity increases, so does their error of the other intensi- ties.

Overall, IPAQ is easy to perform. However, the data that it yields (when compared to objectively collected one) is very inaccurate. That being said, we would not recommend using IPAQ as an independent method for measuring physical activity.

\section{ACKNOWLEDGEMENT}

Article conducted as a part of State funded project № ASDC - 11/15.02.2018

\section{REFERENCES}

Craig CL, Marshall AL, Sjöström M, Bauman

AE, Booth ML, Ainsworth BE, Pratt M, Ekelund U, Yngve A, Sallis JF, Oja P. (2003). International physical activity questionnaire: 12 -country reliability and validity. PubMed, 35(8):1381-95, available at:

https://www.ncbi.nlm.nih.gov/pubmed/12900694 (accessed 8 April 2019)

Hagströmer M, Trost SG, Sjöström M, Berrigan D. (2010). Levels and patterns of objectively assessed physical activity - a comparison between Sweden and the United States. PubMed. May 15;171(10):1055-

64, available at: https://www.ncbi.nlm.nih.gov/ pubmed/20406758 (accessed 10 April 2019)

WHO (2005). Work programme 2005 / 2006: summary, WHO Press, available at: http://www.euro.who.int/ data/assets/pdf_file/0014/101723/execsum_work_prog. pdf?ua $=1$

(accessed 10 March 2019)

WHO (2009). Global health risks. Mortality and burden of disease attributable to selected major risks, WHO Press, available at: https://www.who.int/healthinfo/ global_burden_disease/GlobalHealthRisks_report_full. pdf

(accessed 5 March 2019)

WHO (2010). Global Recommendations on Physical Activity for Health, WHO Press, available at:

https://apps.who.int/iris/bitstream/handle/10665/44399/9789241599979_eng.pdf;jsessionid=81A1E3D27D3B984AD5BDE2A7B3F0799B?sequence $=1$ (accessed 8 April 2019)

\section{Corresponding author:} Jordan Donev, PhD

Associate professor, Department Water sports National Sports Academy „Vassil Levski” Studentski grad, 21, Acad. Stefan Mladenov str. Sofia 1700, Bulgaria E-mail: jordandonev@abv.bg 